DANEEN WARDROP

\title{
IRIDESCENT PANEL WITH GREEN CRENELLATIONS
}

The ant doesn't

detour this clump, goes

right over, muscularity,

waterbag blood

of it, shiny lobes,

six hooves

of it, chomping-

I could list

to this task, tie a look

150

to the dogged

scrambling, but

a pin-star starts

to push the sun aside

and how to count

that rhythm?

I suppose you could say

fireflies-dab

dab-flame-

whip across

a spangled grass.

But ant, gone now, but fireflies, 
doused.

But

I am

afraid 